\title{
Experiment to evaluate the feasibility of the Smart Disc concept
}

\author{
J. Holterman, T.J.A. de Vries, M.P. Koster
}

\author{
presented at the \\ $6^{\text {th }}$ UK Mechatronics Forum International Conference \\ Mechatronics' '98 \\ Skövde, Sweden, 9-11 September 1998
}

published in

J. Adolfsson and J. Karlsén (editors), (1998), Proc. $6^{\text {th }}$ UK Mechatronics Forum International Conference Mechatronics '98, Skövde, Sweden, pp. 217-222 


\title{
Experiment to evaluate the feasibility of the Smart Disc concept
}

\author{
J. Holterman, T.J.A. de Vries, M.P. Koster
}

Cornelis J. Drebbel Institute for Systems Engineering

Department of Electrical Engineering, University of Twente

P.O. Box 217, 7500 AE Enschede, The Netherlands

Phone: +31-53 48927 88, Fax: +31-53 4892223

E-mail: mechatronics@rt.el.utwente.nl,www-address: http://www.rt.el.utwente.nl/mechatronics

Keywords: mechatronics, vibration control, flexible frames, piezo actuation, piezo sensing

Performance of contemporary high-tech equipment is more and more determined by accuracy. Positional deviations due to high frequent vibrations in the floor can be suppressed by mounting the equipment on a frame that acts as a low-pass filter, but low frequent deviations and deviations due to motions inside the machine can only be reduced by increasing the machine frame stiffness. When only passive elements are used during design, large stiffness can only be attained with rigid bodies, which often exhibit bad dynamic behaviour.

In case a machine should be both accurate and fast, a design with active elements should be considered. The Smart Disc is an example of such an active element. It integrates a piezoelectric sensor, a piezoelectric actuator and control electronics, such that it expands when it experiences a compressing force, and vice versa. Insertion of Smart Discs at discrete locations in a machine might compensate for the lack of frame stiffness.

To evaluate the feasibility of the Smart Disc concept an experimental set-up has been designed. Though only one prototype Smart Disc is utilised, the set-up resembles a large class of machines suffering from deviations caused by the non-infinite frame stiffness and non-filtered vibrations in the floor. Experiments with this set-up show a reduction of the positional error of interest in the order of $20 \mathrm{~dB}$ in a frequency range of 0.1 to $10 \mathrm{~Hz}$.

\section{INTRODUCTION}

A machine frame has two main functions. First, it takes care of the relative positioning of machine parts, and second, it provides supporting forces in order to carry the load of machine parts. As the desired accuracy of operation of machines increases, these two functions become hard to combine. The deflection of the machine frame due to load carrying will be of the same order of magnitude as the allowed positioning errors.

When only passive elements are used during design, the above-mentioned problem can only be solved with large stiffness. Large stiffness however can only be attained with rigid bodies, which often exhibit bad dynamic behaviour. Therefore 'designing', with passive elements only, is searching for a compromise between accuracy and speed.
Adequate performance may then not always be attainable for acceptable costs. In that case the use of active elements can help to increase performance or reduce costs.

This paper describes the design and the results of an experiment that may prove the feasibility of an 'active damping' concept with so-called Smart Discs. A Smart Disc is thought of as an integrated device that expands when it experiences a compressing force, and shrinks when it experiences a stretching force. This 'recalcitrant' behaviour can be realised by integration of a piezoelectric sensor, a piezoelectric actuator and control electronics. Insertion of Smart Discs at discrete locations in a machine might compensate for the lack of stiffness of the frame, i.e., reduce positional deviations at certain other locations of interest in the machine. 
The number of applications one can imagine for a Smart Disc-like device is quite large. Nevertheless in this paper only a special class of applications is considered. The main function of the equipment this class covers, is accurate positioning of the top of a non-rigid frame with respect to the base of the frame, as will be explained in the next section. The subsequent sections describe the design of a suitable yet simple experimental set-up, as well as the results of experiments with this set-up.

\section{PROBLEM DESCRIPTION}

To evaluate the feasibility of the Smart Disc concept, this paper concentrates on a special class of applications. This class covers accurate high-tech equipment like electron microscopes, wafersteppers and telescopes. The main function of the frame of these machines is accurate positioning of the top of the frame with respect to the base of the frame.

Usually accurate equipment like this is mounted on a resiliently supported frame that filters the vibrations from the 'fixed' world. As the equipment is most sensitive for high frequent disturbances, this mass-spring configuration, acting as a low-pass filter, is rather appropriate. However, due to the limited filter bandwidth, the top of the frame will still suffer from some low frequent deflection (Fig. 1a).
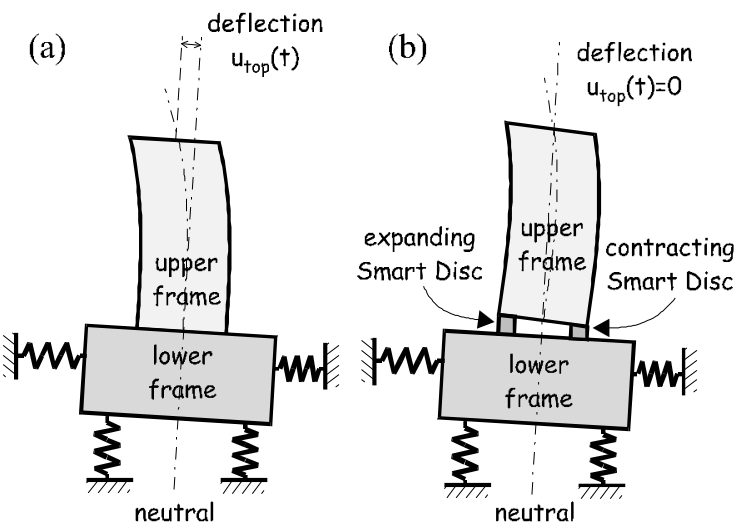

Figure 1. a) Resiliently supported frame.

b) Smart Discs compensate for deviation.

Until now the low frequent disturbances, for instance lower than $10 \mathrm{~Hz}$, hardly affected the performance of the equipment. But with increasing accuracy demands these disturbances can no longer be ignored. One should either reduce the disturbances, by decreasing the cut-off frequency of the low-pass filter, or reduce the effect of the disturbances on the equipment, by increasing the frame rigidity. Both solutions are based on the optimal design of passive elements and may not be satisfying. In that case the use of active elements, Smart Discs for instance, should be considered.

\subsection{Smart Disc concept}

For a good understanding of the Smart Disc concept, it is important to note that the deviation relevant for accurate operation is not the movement of the complete machine (heavy lower frame together with flexible upper frame), but the relative movement between the upper frame and the lower frame, as indicated in Fig. 1. The proposed solution with active elements is illustrated in Fig. 1b. In this planar perception the upper frame is connected to the lower frame by means of two Smart Discs. In a threedimensional configuration, in which a frame can deflect in more than one direction, the frame should be supported by at least three Smart Discs.

The Smart Disc concept in essence boils down to the following. The actuators of the Smart Discs can compensate for the deformations in the upper frame through rotating the base of the frame. Appropriate expansion respectively contraction of the Smart Discs can accomplish the desired amount of rotation.

When one, for instance for economic reasons, insists on a spatially integrated single device, the deformation has to be estimated with the use of information from the sensors of the Smart Discs. If only the lowest mode of vibration is considered, the deflection at the top can be estimated from the load between the upper and the lower frame. This estimation is only possible if each Smart Disc is equipped with knowledge of the actual set-up it is utilised in, such that the control electronics can calculate the necessary amount of expansion or contraction.

An envisioned realisation of the integration of the three main elements of the Smart Disc (piezoelectric sensor, piezoelectric actuator, control electronics) is depicted in Fig. 2.

\section{EXPERIMENT DESIGN}

The Smart Disc concept for the class of applications considered in this paper is based on a rotation of the base of the upper frame in order to 
reduce the positional error at the top of the frame. To experimentally evaluate the feasibility of this concept, it suffices to design a set-up in which only one prototype Smart Disc is used, as will be made clear in this section.

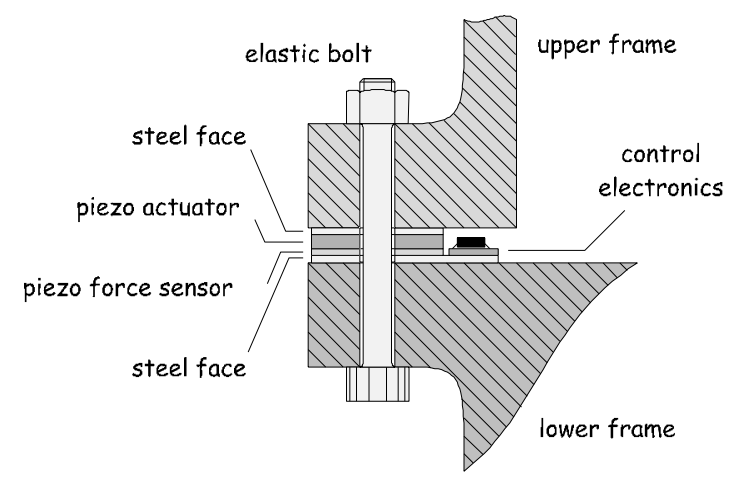

Figure 2. Envisioned realisation of the Smart Disc.

\subsection{Positional error caused by force on top}

The majority of the deflection $u_{t o p}$ in Fig. 1 is due to the lowest mode of vibration of the frame. An approximate model to analyse this is given by a discrete mass and a discrete stiffness, both at the top of a rigid massless frame (Fig. 3). Herewith only the lowest eigenfrequency of the frame is captured. (a)

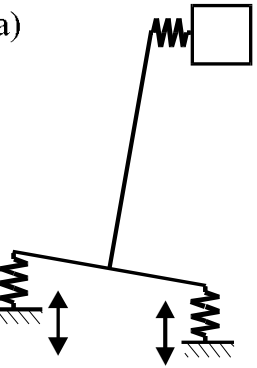

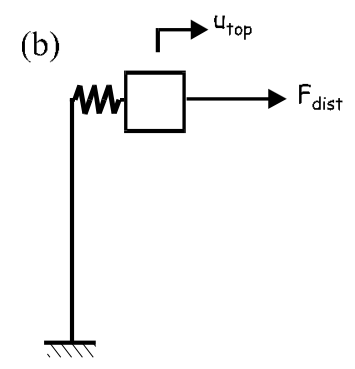

Figure 3. Approximate model of a non-rigid frame, the deformation of which is caused by a) vibrations in the floor,

b) an external force on top.

From the approximate model it can be seen that when the floor suffers from vibrations, the rigid massless frame will rotate or translate. As a consequence the spring at the top of the frame will expand or contract, due to the inertia of the mass it is connected to, and in turn this mass will experience a resulting force (Fig. 3a). Note that in this model the amount of expansion or compression of the spring represents the positional deviation of interest.

From the above considerations it follows that the deviation at the top of the non-rigid frame can be thought of as the effect of a resulting force on the top of the frame. Vibrations in the 'fixed' world thus cause the same kind of positional deviations as an external disturbing force on the top of a non-rigid frame in a non-moving world. Therefore, to evaluate the feasibility of the Smart Disc concept, it suffices to consider a frame in a non-moving world upon which a disturbing force acts at the top (Fig. 3b).

\subsection{Fixed rotation point}

The deviation at the top of the upper frame can be reduced or even completely compensated for by rotation of the base of the upper frame as illustrated in Fig. 1b. The rotation of the base can be accomplished by appropriate expansion respectively compression of the two Smart Discs.

In order to keep the same average height, independent of the angle of rotation, the thickness variation of both Smart Discs must be the same. In that case a fictitious rotation point halfway the two Smart Discs can be defined (Fig. 4a). Therefore, to evaluate the feasibility of the Smart Disc concept, it suffices to implement a physical point of rotation (the hinge point) and only one prototype Smart Disc in the experimental set-up instead of two (Fig. 4b).
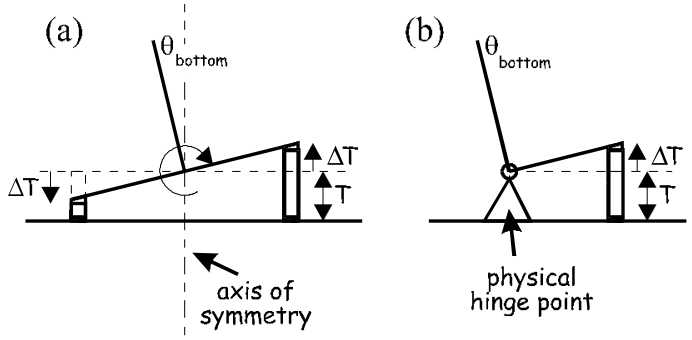

Figure 4. a) Symmetry in the set-up. b) One Smart Disc instead of two.

\subsection{Experimental set-up}

With the previous considerations in mind, the experimental set-up has been designed (Fig. 5). The machine frame is modelled by a simple beam of steel with finite stiffness (dimensions: $200 \times 50 \times 10 \mathrm{~mm}^{3}$ ). The hinge point in the lower corner of the frame (a so-called 'gatscharnier'; Koster, 1998) allows only for a small, but sufficiently large amount of rotation. 
This implementation of the hinge point indeed constitutes a fixed rotation point, as it is hysteresisfree.

The disturbing force is generated by an electromagnet, which is mounted on a separate beam. By the choice of an electromagnet the disturbing force can easily be controlled.

To be able to evaluate the effect of the Smart Disc action, the deflection of the top of the frame should be measured. For this purpose a linear variable differential transformer (LVDT) is mounted on a separate beam. Note that this additional measurement is not used in the control action of the Smart Disc actuator.

In the experimental set-up use is made of a prototype Smart Disc in which a personal computer takes the place of the control electronics. The prototype Smart Disc is treated in the next section.

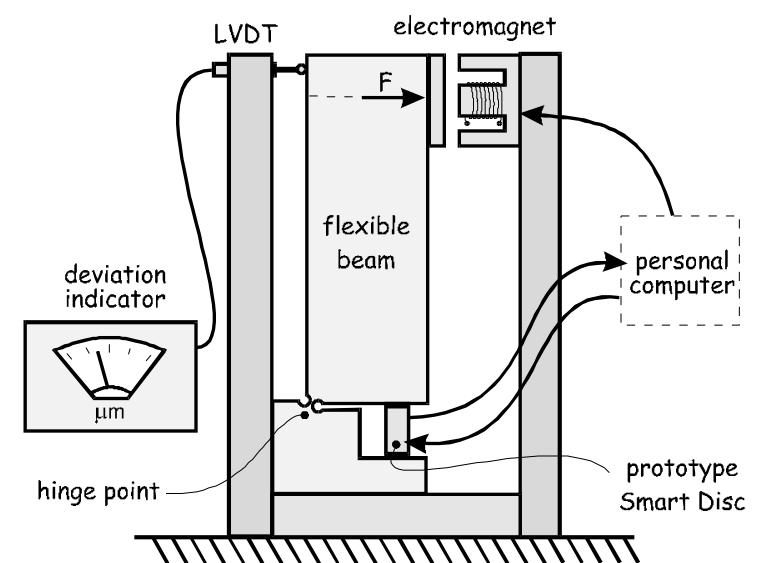

Figure 5. Schematic view of the experimental set-up.

\section{SMART DISC}

As the prototype Smart Disc used in the experimental set-up has not yet been equipped with control electronics, the Smart Disc only integrates an actuator and a sensor. For both the actuator and the sensor use is made of piezoelectric material known as CMA (ceramic multilayer actuator; refer e.g. Miu, 1993), referred to in this paper as piezo mono-stacks.

The prototype Smart Disc consists of two compound piezo stacks (height: $8.4 \mathrm{~mm}$ ), which in turn both consist of three piezo mono-stacks (dimensions: $7 \times 7 \times 2 \mathrm{~mm}^{3}$; see Fig. 6). Two of the mono-stacks are used for actuation and the other is used for sensing. Though in theory it is possible to use only one piezo mono-stack as both a sensor and an actuator, in practice the voltage to be measured ( $1.5 \mathrm{~V})$ would be disturbed too much by the actuating voltage ( $60 \mathrm{~V}$ maximum).
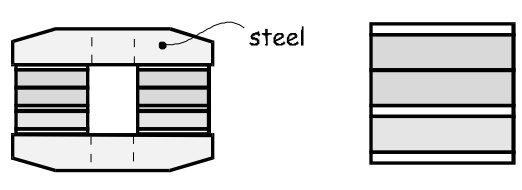

isolator

2 piezo actuators

isolator

piezo sensor

isolator

Figure 6. Prototype Smart Disc (left), built up of two compound piezo stacks (right).

From the dimensions of the piezoelectric layers the piezo mono-stacks consist of (thickness: $20 \mu \mathrm{m}$ ), it can be deduced that the lowest possible resonance frequency of the piezo stacks is above $50 \mathrm{kHz}$ and thus far above the bandwidth relevant to the experiment. Therefore it is reasonable to neglect the dynamic behaviour of a piezo and only to use a quasistatic model (Geraeds, 1996b). A single piezo monostack with $\mathrm{d}_{33}$-coupling (i.e., the direction in which the piezo extends is parallel with the poling axis) can then be described with the following matrix equation:

$$
\left[\begin{array}{c}
\Delta t_{h} \\
\Delta Q
\end{array}\right]=\left[\begin{array}{cc}
C_{\text {mech }} & d \\
d & C_{\text {elec }}
\end{array}\right] \cdot\left[\begin{array}{c}
F_{3} \\
U
\end{array}\right],
$$

with

$\Delta t_{h}: \quad$ thickness variation of the piezo [m],

$\Delta Q:$ excess charge on the piezo [C],

$F_{3}$ : force applied to the piezo [N],

$U: \quad$ voltage across the piezo [V],

and

$C_{\text {mech }}$ : mechanical compliance of the piezo $[\mathrm{m} / \mathrm{N}]$,

$C_{\text {elec }}$ : electrical capacity of the piezo $[\mathrm{C} / \mathrm{V}]$,

$d: \quad$ piezoelectric charge constant $[\mathrm{C} / \mathrm{N}] \equiv[\mathrm{m} / \mathrm{V}]$.

From eq. (1) it can be seen that, due to the piezoelectric coupling phenomenon $(d)$, a piezo can be used both as force sensor and as position actuator. The maximum thickness variation of the single monostacks used in the prototype Smart Disc, when the maximum allowed voltage $(60 \mathrm{~V})$ was applied, was $2.42 \mu \mathrm{m}$. 


\subsection{Force sensor}

When a piezo is used as a force sensor, two designs are possible. The first design is based on zero voltage across, i.e. short-circuiting of the two conducting layers of the piezo. The current through the short circuit then is a measure for the time derivative of the applied force. The second design is based on zero current between the two conducting layers. In this case the voltage across the piezo is a measure for the applied force. As in the latter design the measured signal needs no integration, the piezo force sensor is implemented as a voltage generator.

In the voltage generator configuration, the resistance between the conducting layers is near infinity. As a consequence the initial charge on the piezo is undefined and will drift as a result of external electric disturbances. To prevent malfunctioning of the sensor, the initial charge is forced to zero, by connecting a known shunt resistance parallel to the piezo.

Furthermore, instead of an ideal voltage meter, a non-inverting amplifier with an input resistance near infinity is used. The implementation of the force sensor is shown in Fig. 7. Here the piezo is modelled as a voltage source in series with a capacitor. The transfer function from voltage across the piezo to measured voltage now can be given by

$$
U_{\text {out }}(j \omega)=K \frac{j \omega}{j \omega+\omega_{0}} \cdot U_{\text {piezo }}(j \omega),
$$

in which we recognise a high-pass filter with gain

$$
K=1+\frac{R_{2}}{R_{1}} \text {. }
$$

An important consequence of this implementation is that the force sensor does not operate for frequencies below the cut-off frequency

$$
\omega_{0}=\left(C_{\text {elec }} R_{\text {shunt }}\right)^{-1} \text {. }
$$

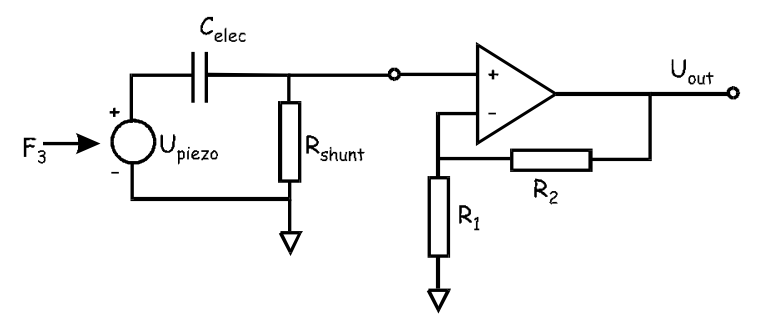

Figure 7. Implementation of the force sensor.
In the experiments use was made of a force sensor implemented according to Fig. 7 with cut-off frequency $0.13 \mathrm{rad} / \mathrm{s}$.

\subsection{Position actuator}

When a piezo is used as a positional actuator, again two designs are possible. Usually a design based on voltage control is used because of its simple implementation. The thickness variation of a mechanically unloaded piezo then is linearly dependent to the applied voltage. An important disadvantage of voltage control, 'hysteresis', is almost completely solved when using the other possible design, based on charge control (Main et al., 1995). Here the thickness variation of the mechanically unloaded piezo is linearly dependent to the applied charge. Though the amplifier needed for charge control is more complex, in the experimental set-up use is made of a charge controlled position actuator.

\subsection{Controller design}

When the dynamics of the experimental set-up are neglected, the set-up can be represented as a multivariable model with only simple gain factors.

$\left[\begin{array}{c}u_{\text {top }} \\ U_{\text {piezo }}\end{array}\right]=\left[\begin{array}{ll}k_{1} & k_{2} \\ k_{3} & k_{4}\end{array}\right] \cdot\left[\begin{array}{c}F_{\text {ext }} \\ U_{\text {act }}\end{array}\right]$

Here we recognise

$u_{t o p}$ : deviation to be compensated for [m],

$U_{\text {piezo }}$ : voltage across the force sensor piezo [V],

$F_{\text {ext }}:$ external force at the top of the frame $[\mathrm{N}]$,

$U_{a c t}:$ voltage across the position actuator piezo [V], and

$k_{1}$ : set-up overall compliance, $0.467 \cdot 10^{-6}[\mathrm{~m} / \mathrm{N}]$,

$k_{2}:$ piezo actuator constant, $-6.22 \cdot 10^{-6}[\mathrm{~m} / \mathrm{V}]$,

$k_{3}$ : piezo sensor constant, $0.15[\mathrm{~V} / \mathrm{N}]$,

$k_{4}$ : piezo cross-talk constant, $1.95[-]$.

The values for the four gain factors have been determined experimentally.

With the use of the simple static model a rather straightforward model-based controller has been designed. This has been motivated by the fact that the emphasis in this experiment was not on controller design but on the evaluation of the feasibility of the Smart Disc concept. 


\section{RESULTS}

With the set-up designed as described in section 3 and 4, two experiments have been performed. The purpose of the first experiment was to determine the frequency domain damping characteristics of the prototype Smart Disc. A sinusoid disturbance force, with amplitude $25 \mathrm{~N}$ was applied to the set-up, in a frequency range from 0.01 to $7 \mathrm{~Hz}$. In case of no active damping this caused a sinusoidal deviation with amplitude $12.5 \mu \mathrm{m}$. The damping the utilisation of the prototype Smart Disc yielded is depicted in Fig. 8.

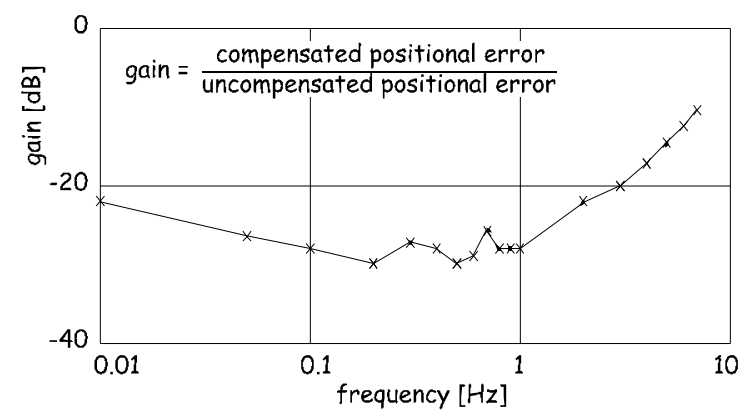

Figure 8. Frequency response of the active damping.

In the second experiment the response of the setup was evaluated in the time-domain. Again the setup with active damping was compared to the uncompensated set-up. The applied force was a $2 \mathrm{~Hz}$ block-shaped wave, resembling deformations due to non-filtered motions inside the machine, thus cursed with high frequent components. From the results (Fig. 9) it can be seen that the Smart Disc indeed is able to compensate for low frequent deviations. However, high frequent disturbances are hardly suppressed.

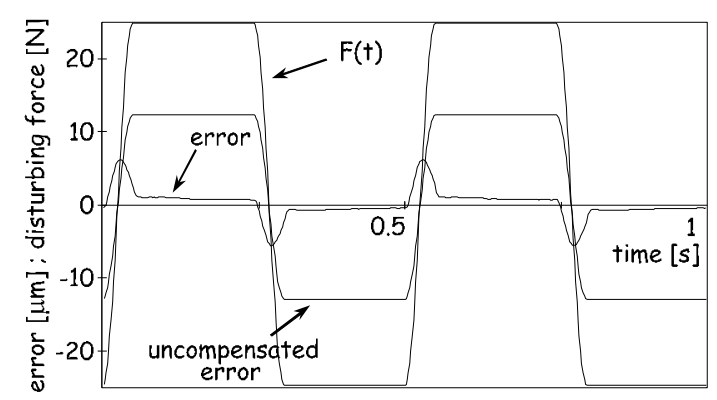

Figure 9. Response to a block wave shaped force.

\section{CONCLUSION}

To study the principle functionality of a Smart Disc, it suffices to have a set-up in which a disturbance force acts on the top of a non-rigid beam with a hinge point in one lower corner and a Smart Disc supporting the other lower corner (Fig. 5).

Experiments with the set-up show that the Smart Disc can compensate for a positional deviation at the top of the upper frame. Within the given disturbance bandwidth of $0.1 \mathrm{~Hz}$ up to $10 \mathrm{~Hz}$ the Smart Disc can realise an attenuation of approximately 10 to $30 \mathrm{~dB}$. The remaining disturbances mainly originate from a higher harmonic cross-talk between the steered actuator voltage and the measured sensor voltage.

These initial results suggest that the concept may be feasible in reality.

\section{ACKNOWLEDGEMENT}

The authors wish to thank Pascal Geraeds; the material for this paper has mainly been taken from his M.Sc. thesis (Geraeds, 1996a).

\section{REFERENCES}

Geraeds, P.M.J., (1996a), Active damping of low frequent vibrations in non-infinite stiff frames with a Smart Disc (an experiment design), M.Sc. thesis, Report no 029R96, Control Laboratory, Electrical Engineering Department, University of Twente, Enschede, The Netherlands.

Geraeds, P.M.J., (1996b), Overview of piezoelectric ceramics, Report no 034R96, Control Laboratory, Electrical Engineering Department, University of Twente, Enschede, The Netherlands.

Koster, M.P., (1998), Constructieprincipes voor het nauwkeurig bewegen en positioneren (in Dutch), Twente University Press, Enschede, The Netherlands.

Main, J.A., E. Garcia, D.V. Newton, (1995), Precision Position Control of Piezoelectric Actuators Using Charge Feedback, Journal of Guidance, Control and Dynamics, Vol. 18, No. 5, September-October, pp. 1068-1073.

Miu, D.K., (1993), Mechatronics, Electromechanics, Electromechanics and Contromechanics, Department of Mechanical, Aerospace and Nuclear Engineering, University of California, Los Angeles, Springer Verlag New York, USA. 